\title{
Editorial
}

\section{Stratospheric Processes and Their Role in Climate}

\author{
JianJun Xu, ${ }^{1,2}$ K. Mohanakumar, ${ }^{3}$ Dong Guo, ${ }^{4}$ Yu Liu, ${ }^{5}$ and Jia Yue ${ }^{6}$ \\ ${ }^{1}$ College of Ocean and Meteorology, Guangdong Ocean University, Zhanjiang, China \\ ${ }^{2}$ George Mason University, Fairfax, VA, USA \\ ${ }^{3}$ Cochin University of Science and Technology, Cochin, India \\ ${ }^{4}$ Key Laboratory of Meteorological Disaster, Ministry of Education/Joint International Research Laboratory of Climate and \\ Environment Change/Collaborative Innovation Center on Forecast and Evaluation of Meteorological Disasters, Nanjing University of \\ Information Science and Technology, Nanjing, China \\ ${ }^{5}$ Chinese Academy of Meteorological Sciences, Beijing, China \\ ${ }^{6}$ Hampton University, Hampton, VA, USA
}

Correspondence should be addressed to JianJun Xu; jxu14@gmu.edu and Dong Guo; dongguo@nuist.edu.cn

Received 30 October 2017; Accepted 31 October 2017; Published 20 November 2017

Copyright (C) 2017 JianJun Xu et al. This is an open access article distributed under the Creative Commons Attribution License, which permits unrestricted use, distribution, and reproduction in any medium, provided the original work is properly cited.

The stratosphere is the transition region which interacts with the climate systems in the lower atmosphere and the richly ionized upper atmosphere. Therefore, this part of the atmosphere involves a long list of challenging scientific problems of basic research, including its thermal structure, energetics, composition, dynamics, chemistry, and their roles in regulating the climate of Earth. In this special issue, we have gathered a few papers that address such issues.

Spatial and temporal distribution variation mechanisms of atmospheric composition over the Tibetan Plateau and in polar regions were analyzed. The linkage between polar vortex and ozone depletion in the Antarctic stratosphere during the period 1979-2016 was investigated, and the possible mechanism for their relationship is also discussed. Moreover, the contributions of energetic particle precipitation and solar UV emission to ozone changes in the polar upper atmosphere were compared. Furthermore, local climate effects of the Tibetan Plateau Ozone Valley were investigated by numerical simulations using Community Atmosphere Model version 5.1.1. In addition, the characteristics of water vapor in the UTLS over the Tibetan Plateau were analyzed by using AURA/MLS satellite data and ERA-Interim reanalysis datasets.

Dynamic processes such as wave and circulation were investigated in the papers. Models with well-resolved stratospheres in the CMIP5 models simulated stratospheric Northern Hemisphere polar vortex better, because the models include better dynamic processes. And the role of RossbyWave propagation in a North American extreme cold event in January 2014 was analyzed by calculating the Eliassen-Palm flux and Plumb wave activity flux by using the European Centre for Medium-Range Weather Forecasts interim reanalysis daily dataset. Moreover, based on data from 16 chemistry-climate models (CCMs) and separate experimental results using a state-of-the-art CCM, the trends in the Brewer-Dobson circulation during the second half of the 20th century and the first half of the 21st century were examined. Furthermore, mass transport during the east-west oscillation of the Asian Summer Monsoon Anticyclone was analyzed.

In addition to dynamic and chemical processes, radiation processes are also important. Chebyshev and Legendre polynomial expansions of phase function of cloud and aerosol particles are compared, and a novel automatic contrail detecting method based on Himawari-8 stationary satellite imagery was proposed. Moreover, warming and ENSO signals in the upper stratosphere and lower troposphere were studied.

JianJun Xu

K. Mohanakumar

Dong Guo

Yu Liu

Jia Yue 

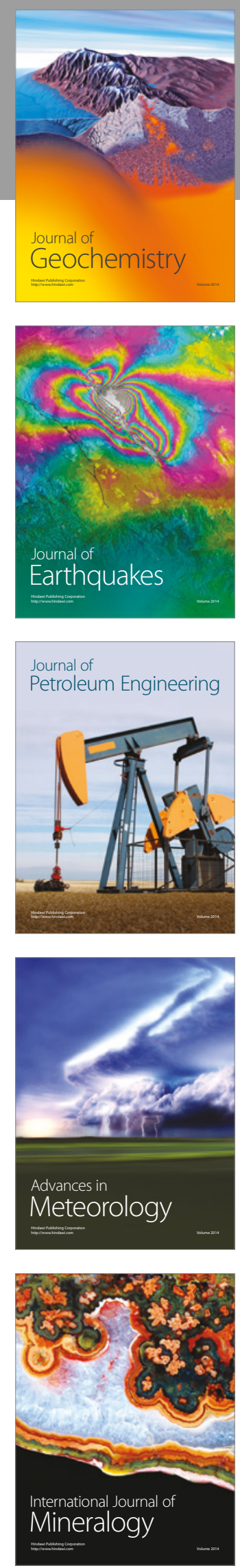
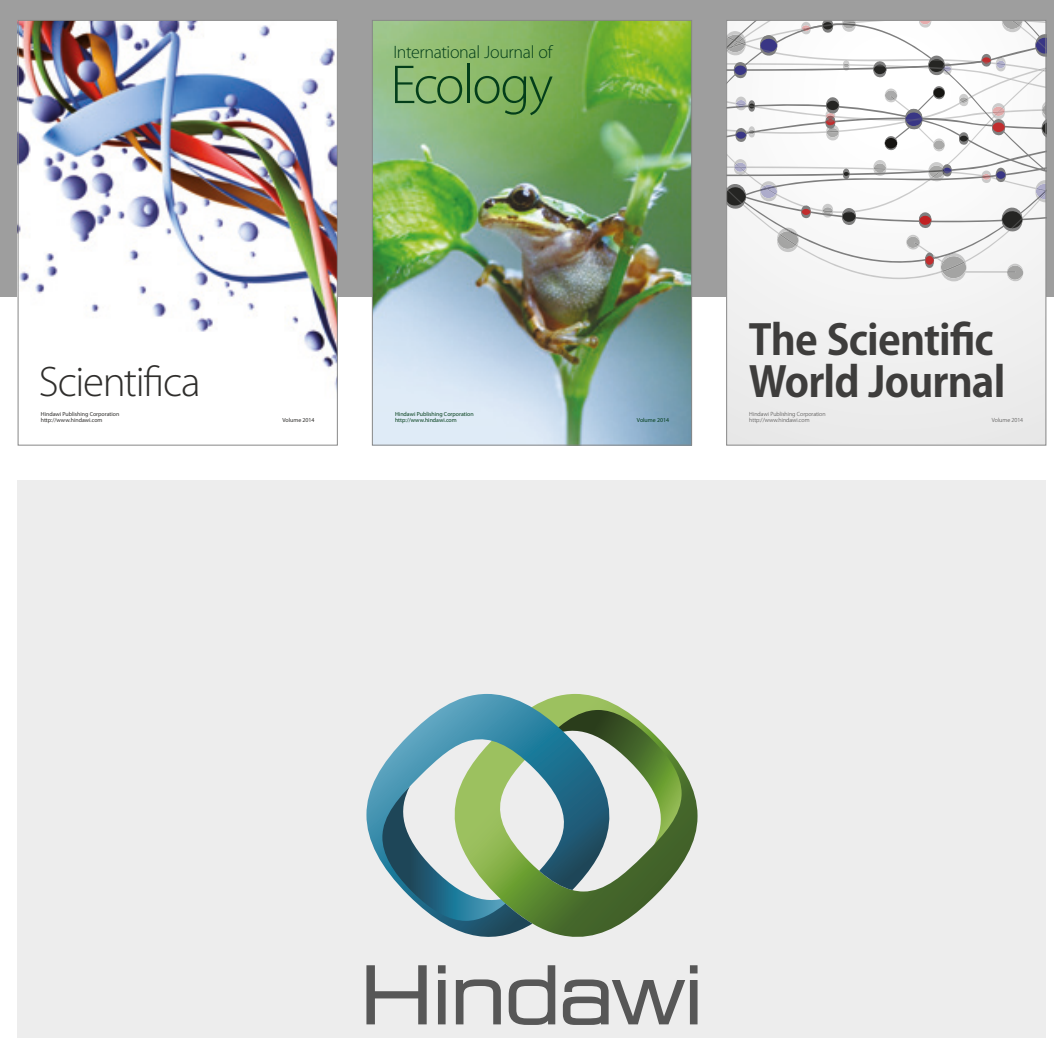

Submit your manuscripts at

https://www.hindawi.com
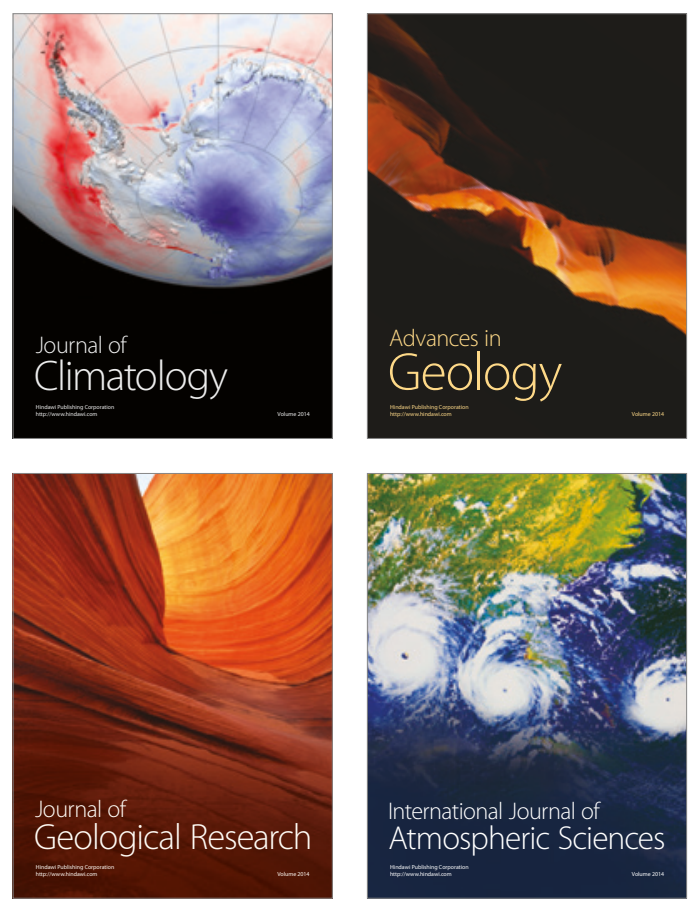

The Scientific

World Journal
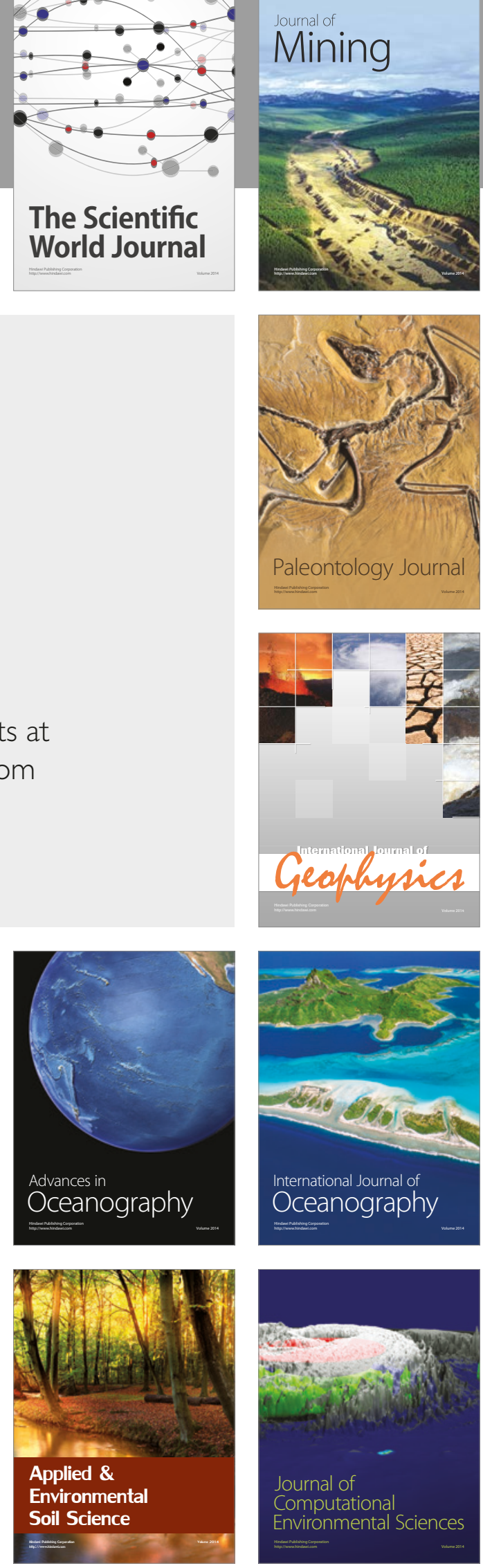